\title{
Infantile epileptic-dyskinetic encephalopathy
}

INSERM

\section{Source}

INSERM. (1999). Orphanet: an online rare disease and orphan drug data base. Infantile epileptic-dyskinetic encephalopathy. ORPHA:364063

Infantile epileptic-dyskinetic encephalopathy is a monogenic disease with epilepsy characterized by developmental delay and infantile spasms in the first months of life, followed by chorea and generalized dystonia and progressing to quadriplegic dyskinesia, recurrent status dystonicus, intractable focal epilepsy and severe intellectual disability. 